\title{
Syntheses, X-ray Crystal Structure Analyses, and Solid-State NMR Studies of Some Zwitterionic Organofluorosilicates
}

\author{
Reinhold Tacke, "† Joachim Becht, ${ }^{\dagger}$ Angel Lopez-Mras, ${ }^{\dagger}$ William S. Sheldrick, ${ }^{\ddagger}$ and Angelika Sebald! \\ Institut für Anorganische Chemie, Universität Karlsruhe, Engesserstrasse, Geb. 30.45, \\ D-7500 Karlsruhe, Germany, Lehrstuhl für Analytische Chemie, Ruhr-Universităt Bochum, \\ Universitåtsstrasse 150, D-4630 Bochum, Germany, and Bayerisches Geoinstitut, Universităt Bayreuth, \\ Postfach 101251, D-8580 Bayreuth, Germany
}

Received December 29, 1992

\begin{abstract}
Reaction of dimethoxy(methyl)(pyrrolidinomethyl)silane [Me(MeO) ${ }_{2} \mathrm{SiCH}_{2} \mathrm{NC}_{4} \mathrm{H}_{8}, \mathrm{NC}_{4} \mathrm{H}_{8}=$ pyrrolidino] and methoxy (methyl)phenyl(pyrrolidinomethyl)silane [MePh(MeO)SiCH$\left.{ }_{2} \mathrm{NC}_{4} \mathrm{H}_{8}\right]$ with hydrogen fluoride at $0^{\circ} \mathrm{C}$ in ethanol/hydrofluoric acid yielded trifluoro(methyl)(pyrrolidiniomethyl)silicate [ $\mathrm{F} 3 \mathrm{Si}(\mathrm{Me}) \mathrm{CH}_{2} \mathrm{~N}(\mathrm{H}) \mathrm{C}_{4} \mathrm{H}_{8}(9), \mathrm{N}(\mathrm{H})$ $\mathrm{C}_{4} \mathrm{H}_{3}=$ pyrrolidinio] (yield $90 \%$ and $89 \%$, respectively). The related trifluoro(phenyl)(pyrrolidiniomethyl)silicate $\left[\mathrm{F}_{3} \mathrm{Si}(\mathrm{Ph}) \mathrm{CH}_{2} \mathrm{~N}(\mathrm{H}) \mathrm{C}_{4} \mathrm{H}_{8}(10)\right]$ was obtained by reaction of dimethoxy(phenyl)(pyrrolidinomethyl)silane [Ph$(\mathrm{MeO})_{2} \mathrm{SiCH}_{2} \mathrm{NC}_{4} \mathrm{H}_{8}$ ] with $\mathrm{HF}$ (yield $81 \%$ ). 9 and 10 are the first zwitterionic (ammonioalkyl)organotrifluorosilicates. Crystal data for these two compounds are as follows. 9: space group $P Z_{1} / n, a=8.359(3) \AA, b=11.388$ (4) $\AA$, $c=9.076(2) \AA, \beta=93.40(3)^{\circ}, V=862.4(5) \AA^{3}, T=-100^{\circ} \mathrm{C}, Z=4, R=0.037, R_{w}=0.037$. 10: space group $P 2_{1} / n, a=9.542(4) \AA, b=12.536(4) \AA, c=10.996(3) \AA, \beta=114.13(3)^{\circ}, V=1200.4(7) \AA^{3}, T=-100^{\circ} \mathrm{C}, Z$ $=4, R=0.043, R_{w}=0.043$. The zwitterionic organofluorosilicates 9 and 10 contain a pentacoordinate silicon atom (formally negatively charged) and a tetracoordinate nitrogen atom (formally positively charged). In the crystal, the coordination polyhedrons around the silicon atoms can be described as distorted trigonal bipyramids: two of the fluorine atoms occupy the axial sites, whereas the third fluorine atom and the two carbon atoms are found in the equatorial positions. The zwitterionic species also exist in solution $\left(9, \mathrm{CD}_{3} \mathrm{CN}, \mathrm{CDCl}_{3} ; 10, \mathrm{CD}_{3} \mathrm{CN} ;{ }^{1} \mathrm{H},{ }^{13} \mathrm{C}\right.$, and ${ }^{19} \mathrm{~F}$ NMR), displaying a rapid ligand exchange at room temperature (one ${ }^{19} \mathrm{~F}$ resonance). Solid-state NMR studies $\left({ }^{15} \mathrm{~N},{ }^{29} \mathrm{Si}\right.$ ) on authentic tetrafluoro(pyrrolidiniomethyl)silicate $\left[\mathrm{F}_{4} \mathrm{SiCH}_{2} \mathrm{~N}\left(\mathrm{H}_{1} \mathrm{C}_{4} \mathrm{H}_{8}(7)\right]\right.$ and on (3ammoniopropyl)tetrafluorosilicate $\left[\mathrm{F}_{4} \mathrm{Si}\left(\mathrm{CH}_{2}\right)_{3} \mathrm{NH}_{3}(8)\right]$ revealed evidence that 8 is indeed the earlier postulated product formed in the reaction of (3-aminopropyl)triethoxysilane [ $(\mathrm{EtO})_{3} \mathrm{Si}\left(\mathrm{CH}_{2}\right)_{3} \mathrm{NH}_{2}$ ] with $\mathrm{HF}$ in ethanol/ hydrofluoric acid $\left(7, \delta\left({ }^{15} \mathrm{~N}\right)=-318.5 \mathrm{ppm}, \delta\left({ }^{29} \mathrm{Si}\right)=-121.1 \mathrm{ppm} ; 8, \delta\left({ }^{15} \mathrm{~N}\right)=-345.3 \mathrm{ppm}, \delta(29 \mathrm{Si})=-112.4 \mathrm{ppm}\right)$.
\end{abstract}

\section{Introduction}

During the last few years, numerous papers on the synthesis and structure of ionic organosilicates have been published. ${ }^{1,2}$ In contrast, only a small number of zwitterionic organosilicates have been described ${ }^{3-10}$ (for a recent review, see ref 11 ). Most of these zwitterions belong to the class of spirocyclic silicates (examples: $1.1 / 4 \mathrm{CH}_{3} \mathrm{CN}, 5,82 \cdot \mathrm{CH}_{3} \mathrm{CN},{ }^{4,8} 3 \cdot \mathrm{CH}_{3} \mathrm{CN}, 6,84,84 \cdot \mathrm{H}_{2} \mathrm{O}, 8,5,{ }^{, 8}$ and $\left.6^{8}\right)$, whereas compound $7^{10}$ represents the only authentic zwitterionic organofluorosilicate that has been characterized by $X$-ray diffraction and NMR-spectroscopic studies (Scheme I). In this context it should be mentioned that the existence of the structurally related zwitterionic (ammonioalkyl)tetrafluorosilicate 8 (Scheme I) was already postulated nearly two decades previously. ${ }^{12}$ As its identity was based only on an elemental analysis, the authenticity of this compound, however, has remained uncertain. We now wish to report the syntheses and crystal structures of the

- To whom correspondence should be addressed.

+ Universitat Karlaruhe.

t Ruhr-Univeraitat Bochum.

Universitut Bayreuth.

(1) Reviews: (a) Tandura, S. N.; Voronkov, M. G.; Alekseev, N. V. Top. Curr. Chem. 1986, 131, 99-189. (b) Voronkov, M. G.; Gubanova, L. I. Main Group Met. Chem. 1987, 10, 209-286. (c) Sheldrick, W.S. In The Chemistry of Organic Silicon Compounds, Part 1; Patai, S., The Chemistry of Organic Silicon Compounds, Part 1; Patai, S., Rappoport, Z., Eds.; Wiley \& Sons: Chichester, England, 1989; pp
227-303. (d) Corriu, R. J.P.; Young, J.C. In The Chemistry of Organic 227-303. (d) Corriu, R. J. P.; Young, J. C. In The Chemistry of Organic
silicon Compounds, Part 2; Patai, S., Rappoport, Z., Eds.; Wiley \& Silicon Compounds, Part 2; Patai, S., Rappoport, Z., Eds.; Wiley \&
Sons: Chichester, England, 1989; pp 1241-1288. (c) Corriu, R. J. P.
J. Organomet. Chem. 1990, $400,81-106$. (f Holmes, R. R. Chem. Rev. J. Organomet. Chem. I990, $400,81-106$. (f) Holmes, R. R. Chem. Rev.
$1990,90,17-31$. (g) Tammo, K.; Hayashi, T.; Ito, K. In Frontiers of Organosilicon Chemistry, Bassindale. A. R., Gaspar, P. P., Eds.; The Royal Society of Chemistry: Cambridse, Ensland, 1991; pp 197-207. (b) Corriv, R. J. P.; Young, J. C. In The Silicon Heteroatom Bond; Patai, S., Rappoport, Z., Eds.; Wiley \& Sons: Chichester, England, 1991; pp 1-66.
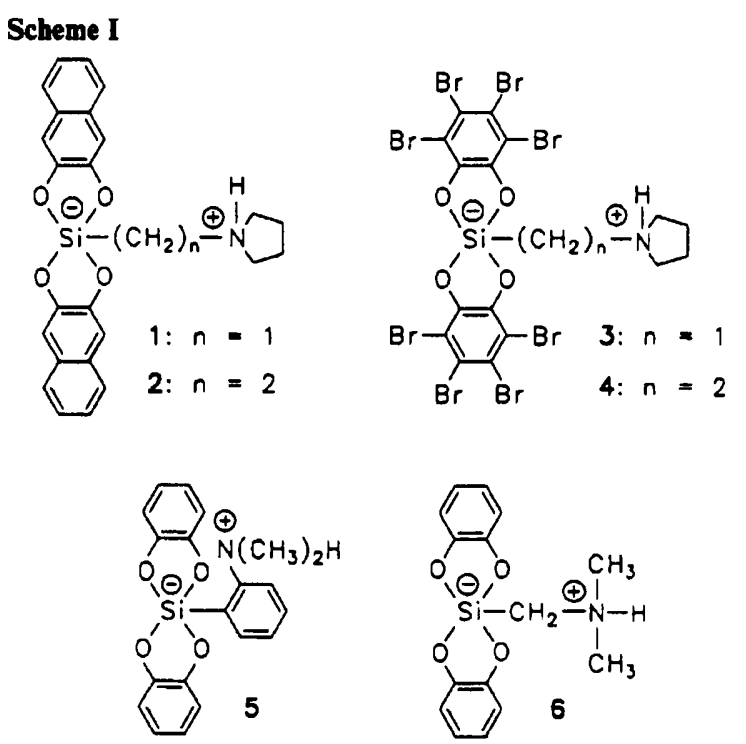

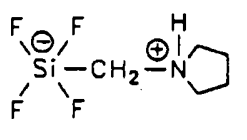

7

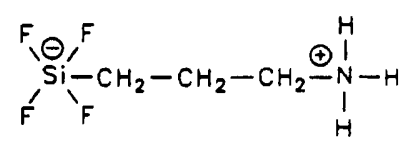

8 first zwitterionic (ammonioalkyl)organotrifluorosilicates 9 and 10 (Scheme II). In addition, the results of solid-state NMR studies on 7, 9, and 10 are described. In order to establish its 
Scheme II

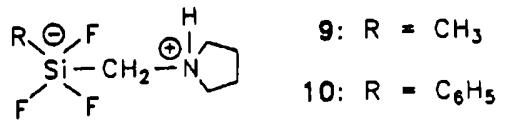

identity unequivocally, compound 8 was resynthesized and included in these investigations.

\section{Experimental Section}

(a) Syntheses. Except for the reactions with hydrofluoric acid, all syntheses were carried out under dry nitrogen. The solvents used were dried according to standard procedures and stored under nitrogen. The reactions with hydrofluoric acid were carried out in polypropylene or Nalgene beakers under normal atmospheric conditions; for filtrations polypropylene suction flasks, polypropylene Buchner funnels, and normal commercial filter paper were used. Melting points were determinated with a Leitz Laborlux S microscope, equipped with a heater (Leitz, Model $\mathrm{M}$ 310). The ${ }^{1} \mathrm{H},{ }^{13} \mathrm{C}$ and ${ }^{19} \mathrm{~F}$ solution-state $N M R$ spectra were recorded on a Bruker AC. 250 spectrometer $\left({ }^{1} \mathrm{H}, 250.1 \mathrm{MHz} ;{ }^{13} \mathrm{C}, 62.9 \mathrm{MHz} ;{ }^{19} \mathrm{~F}\right.$, $235.4 \mathrm{MHz}$ ). The ${ }^{29} \mathrm{Si}$ NMR spectra were recorded with a Bruker WP. $300(59.6 \mathrm{MHz})$ spectrometer. Except for some low-tempera ture studies with 9, all solution-state NMR experiments were carried out at room temperature; $\mathrm{CD}_{3} \mathrm{CN}, \mathrm{CDCl}_{3}$ and $\mathrm{CD}_{3} \mathrm{OD}$ were used as solvents. Chemical shifts (ppm) were determined relative to internal $\mathrm{CHCl},{ }^{1} \mathrm{H}$, $\delta$ 7.25), $\mathrm{CDCl}_{3}\left({ }^{13} \mathrm{C}, \delta 77.05\right), \mathrm{CD}_{3} \mathrm{OH}\left({ }^{1} \mathrm{H}, \delta 4.85\right), \mathrm{CD}_{3} \mathrm{OD}\left({ }^{13} \mathrm{C}, \delta\right.$ 49.0), $\mathrm{CD}_{2} \mathrm{HCN}\left({ }^{1} \mathrm{H}, \delta 1.93\right), \mathrm{CD}_{3} \mathrm{CN}\left({ }^{13} \mathrm{C}, \delta 1.3\right)$, and relative to internal TMS ( $\left.{ }^{29} \mathrm{Si}, \delta 0\right)$ and $\mathrm{CFCl}\left({ }^{19} \mathrm{~F}, \delta 0\right)$. Assignment of the ${ }^{13} \mathrm{CNMR}$ data was supported by DEPT experiments. Mass spectra were obtained with a Varian MAT-711 and a Finnigan MAT-8430 mass spectrometer (EI MS, 70eV; FAB MS, 3-nitrobenzyl alcohol as liquid matrix, xenon as FAB source). The selected $m / z$ values given refer to the isotopes ${ }^{\prime} H$, ${ }^{12} \mathrm{C},{ }^{14} \mathrm{~N},{ }^{16} \mathrm{O},{ }^{19} \mathrm{~F}$, and ${ }^{20} \mathrm{Si}$.

(2) Selected original papers: (a) Frye, C. L. J. Am. Chem. Soc. 1964, 86, 3170-3171. (b) Boer, F. P.; Flynn, J. J.; Turley, J. W. J. Am. Chem. Soc. 1968, 90, 6973-6977. (c) Schomburg, D. J. Organomet. Chem. 1981, 221, 137-141. (d) Holmes, R. R.; Day, R. O.; Harland, J. J.; Sau, A. C.; Holmes, J. M. Organometallics 1984, 3, 341-347. (e) Holmes, R. R.; Day, R. O.; Harland, J.; Holmes, J. M. Organometallics 1984, 3, 347-353. (f) Schombure, D.; Krebs, R. Inorg. Chem. 1984, 23, 13781381. (a) Holmes, R. R. Day, R. O. Chandrasekhar, V. Holmes, J. 1381. (8) Holmes, R. R.; Day, R. O.; Chandrasekhar, V.; Holmes, J. M. Inorg. Chem. 1985, 24, 2009-2015. (h) Holmes, R. R.; Day, R. O.; 24, 2016-2020. (i) Damrauer, R.; Danahey, S. E. Organometallics 1996, 5, 1490-1494. (j) Harland, J. J.; Payne, J. S.; Day, R. O.; Holmes, R. R. Inorg. Chem. 1987, 26, 760-765. (k) Dixon, D. A.; Hertler, W. R.; Bruce Chase, D.; Farnham, W. B.; Devidson, F. Inorg. Chem. 1988, 27, 4012-4018, (1) Kira, M.; Sato, K.; Saturai, H. J. Am. Chem. Soc. 1988, 110, 4599-4602. (m) Johnson, S. E.; Day, R. O.; Holmes, R. R. 1988, 110, 4599-4602. (m) Johnson, S. E.; Day, R. O.; Holmes, R. R.
Inorg. Chem. 1989, 28, 3182-3189. (n) Johnson, S. E.; Payne, J. S.; Inorg. Chem. 1989, 28, 3182-3189. (n) Johnson, S. E.; Payne, J. S.; 3198. (o) Johnson, S. E.; Deiters, J. A.; Day, R. O.; Holmes, R. R. J. Am. Chem. Soc. 1989, 111, 3250-3258. (p) Day, R. O.; Sreelatha, C.; Deiters, J. A. Johnson, S. E.; Holmes, J. M.; Howe, L.; Holmes, R. R. Organometallics 1991, 10, 1758-1766. (q) Laine, R. M.; Youngdabl Organometallics 1s91, 0 , R. Uhm, J. Nature 1991, 353, 642-644. (r) Dettlaff-Weglikowska, U.; Hey-Hawkins, E.; von Schnering, H. G. Z. Naturforsch., B 1991, 46, 609-614. (s) Tamso, K.; Hayashi, T.; Ito, Y.;Shiro, M. Organometallics 1992, 11, 182-191. (t) Tamao, K.; Hayashi, T.; Ito, Y.; Shiro, M. Organometallics 1992, 11, 2099-2114.

(3) Krebs, R. Dissertation, Technical University of Braunschweig, 1987. Schomburg, D.; Krebs, R. Unpublished results.

(4) Strohmann, C.: Tacke, R.; Mattern, G.; Kuhs, W. F. J. Organomet. Chem. 1991, 403, 63-71.

(5) Tacke, R.; Sperlich, J.; Strohmann, C.; Mattern, G. Chem. Ber. 1991, 124, 1491-1496.

(6) Tacke, R.; Sperlich, J.; Strohmann, C.; Frank, B.; Mattern, G. Z. Kristallogr. 1992, 199, 91-98.

(7) Tacke, R.; Wiesenberger, F.; Lopez-Mras, A.; Sperlich, J.; Mattern, G. Z. Naturforsch., B 1992, 47, 1370-1376.

(8) Tacke, R.; Lopez-Mras, A.; Sperlich, J.; Strohmann, C.; Kuhs, W. F.; Mattern, G.; Sebald, A. Chem. Ber. 1993, 126, 851-861.

(9) Tacke, R.; Lopez-Mras, A.; Sheldrick, W.S.; Sebald, A. Z. Anorg. Allg. Chem. 1993, 619, 347-358.

(10) Tacke, R.; Becht, J.; Mattern, G.; Kuhs, W. F. Chem. Ber. 1992, 125, 2015-2018.

(11) Tacke, R.; Bocht, J.; Lopez-Mras, A.; Sperlich, J. J. Organomet. Chem. $1993,446,1-8$

(12) (a) Dathe, C. Dissertation, Technical University of Dresden, 1966. (b) Maller, R. Organomet. Chem. Rev. 1966, 1, 359-377. (c) Müller, R. Z. Chem. 1994, 24, 41-50.
Tetrafluoro(pyrrolidiniomethyl)silicate (7). Synthesis as described in ref 10.

(3-Ammoniopropyl)tetrafluorosilicate (8). A solution of 11 (11.1 8, $50.1 \mathrm{mmol})$ in ethanol $(30 \mathrm{~mL})$ was added dropwise at $0^{\circ} \mathrm{C}$ during $5 \mathrm{~min}$ to a stirred solution of $40 \%$ hydrofluoric acid ( $14.0 \mathrm{~s}, 280 \mathrm{mmol}$ of HF) in ethanol $(20 \mathrm{~mL})$. After $2 \mathrm{~h}$ stirring at $0^{\circ} \mathrm{C}$, the precipitate was filtered off and recrystallized from water to give $6.53 \mathrm{~g}$ (yield 80\%) of tabular, colorless crystals; sublimation at $190^{\circ} \mathrm{C}$. ${ }^{1} \mathrm{H}$ NMR (CD, OD): 80.70 $0.76\left(\mathrm{~m}, 2 \mathrm{H}, \mathrm{SiCH}_{2} \mathrm{C}\right), 1.70-1.82\left(\mathrm{~m}, 2 \mathrm{H}, \mathrm{CCH}_{2} \mathrm{C}\right), 2.85-2.91(\mathrm{~m}, 2$ $\left.\mathrm{H}, \mathrm{NCH}_{2} \mathrm{C}\right) .{ }^{3} \mathrm{C}$ NMR $\left(\mathrm{CD}_{3} \mathrm{OD}\right): \delta 13.2\left(\mathrm{SiCH}_{2} \mathrm{C}\right), 24.3\left(\mathrm{CCH}_{2} \mathrm{C}\right)$, $43.3\left(\mathrm{NCH}_{2} \mathrm{C}\right)$. EI MS: $m / z 143\left(15 \%,[\mathrm{M}-\mathrm{HF}]^{+}\right), 30(100 \%$, $\mathrm{CH}_{2}-\mathrm{NH}_{2}{ }^{+}$). Anal. Calod for $\mathrm{C}_{3} \mathrm{H}, \mathrm{F} 4 \mathrm{NSi}$ : C, 22.08; $\mathrm{H}, 5.56 ; \mathrm{F}, 46.57$; $\mathrm{N}$, 8.58. Found: $\mathrm{C}, 22.2 ; \mathrm{H}, 5.6 ; \mathrm{F}, 46.3 ; \mathrm{N}, 8.6$.

Triflooro(methyl) (pyrrolidiniomethyl) silicate (9). Method A. A solution of $12(2.84 \mathrm{~g}, 15.0 \mathrm{mmol})$ in ethanol $(10 \mathrm{~mL})$ was added dropwise at $0^{\circ} \mathrm{C}$ during $10 \mathrm{~min}$ to a stirred solution of $40 \%$ hydrofluoric acid ( 3.75 8. $75.0 \mathrm{mmol}$ of $\mathrm{HF})$ in ethanol $(10 \mathrm{~mL})$. After $2 \mathrm{~h}$ stirring at $0^{\circ} \mathrm{C}$, the precipitate was filtered off and recrystallized from ethanol to give 2.50 $g$ (yield $90 \%$ ) of prismatic, colorless crystals; sublimating at $115^{\circ} \mathrm{C}$, dec $170^{\circ} \mathrm{C}$. Recrystallization from methanol, acetonitrile and water gave the same results. For analytical data, see below.

Method B. A solution of $15(1.00 \mathrm{~g}, 4.25 \mathrm{mmol})$ in ethanol $(5 \mathrm{~mL})$ was added dropwise at $0^{\circ} \mathrm{C}$ during 5 min to a stirred solution of $40 \%$ hydrofluoric acid $(0.64 \mathrm{~g}, 12.8 \mathrm{mmol}$ of HF) in ethanol $(10 \mathrm{~mL})$. After $2 \mathrm{~h}$ stirring at $0^{\circ} \mathrm{C}$, the precipitate was filtered off and recrystallized from ethanol to give $0.70 \mathrm{~g}$ (yield $89 \%$ ) of prismatic, colorless crystals; sublimating at $115^{\circ} \mathrm{C}$, dec $170^{\circ} \mathrm{C}$. ${ }^{1} \mathrm{H}$ NMR $\left(\mathrm{CD}_{3} \mathrm{CN}\right): \delta-0.01(\mathrm{~s}$, $\left.3 \mathrm{H}, \mathrm{SiCH}_{3}\right), 1.9-2.1\left(\mathrm{~m}, 4 \mathrm{H}, \mathrm{CCH}_{2} \mathrm{C}\right), 2.51\left(\mathrm{~s}, 2 \mathrm{H}, \mathrm{SiCH}_{2} \mathrm{~N}\right), 2.8-3.0$ and 3.5-3.7 (m, $\left.4 \mathrm{H}, \mathrm{NCH}_{2} \mathrm{C}\right), 6.8-7.6$ (broad s, $\left.1 \mathrm{H}, \mathrm{NH}\right)$. ${ }^{13} \mathrm{C} \mathrm{NMR}$ $\left(\mathrm{CD}_{3} \mathrm{CN}\right): \delta 23.7\left(\mathrm{CCH}_{2} \mathrm{C}\right), 50.5\left(\mathrm{SiCH}_{2} \mathrm{~N}\right), 58.0\left(\mathrm{NCH}_{2} \mathrm{C}\right), \mathrm{SiCH}_{3}$ resonance not detectable (overlapping with the reference signal). ${ }^{19} \mathrm{~F}$ NMR $\left(\mathrm{CD}_{3} \mathrm{CN}\right): \delta-102.2$ (broad s). ${ }^{1} \mathrm{H} \mathrm{NMR}\left(\mathrm{CDCl}_{3}\right): \delta 0.13$ (s, 3 $\left.\mathrm{H}, \mathrm{SiCH}_{3}\right), 1.95-2.15\left(\mathrm{~m}, 4 \mathrm{H}, \mathrm{CCH}_{2} \mathrm{C}\right), 2.60\left(\mathrm{~d}, 2 \mathrm{H}, \mathrm{SiCH}_{2} \mathrm{~N}\right), 2.7-2.9$ and $3.7-3.8\left(\mathrm{~m}, 4 \mathrm{H}, \mathrm{NCH}_{2} \mathrm{C}\right), 8.0$ (broad $\mathrm{s}, 1 \mathrm{H}, \mathrm{NH}$ ). ${ }^{13} \mathrm{C}$ NMR $\left(\mathrm{CDCl}_{3}\right): \delta 2.1\left(\mathrm{SiCH}_{3}\right), 23.9\left(\mathrm{CCH}_{2} \mathrm{C}\right), 50.5\left(\mathrm{SiCH}_{2} \mathrm{~N}\right), 58.0\left(\mathrm{NCH}_{2} \mathrm{C}\right)$. ${ }^{19} \mathrm{~F}$ NMR $\left(\mathrm{CDCl}_{3}, 20^{\circ} \mathrm{C}\right.$ ): $\delta-106.0$ (broad s; $\nu_{1 / 2} \approx 1995 \mathrm{~Hz}$ ). ${ }^{19} \mathrm{~F}$ NMR $\left(\mathrm{CDCl}_{3,}-70^{\circ} \mathrm{C}\right) ; \delta-91.5\left(\mathrm{~s}, 2 \mathrm{~F}_{\mathrm{ux}} J_{\mathrm{SIF}(\mathrm{ux})}=242.9 \mathrm{~Hz}\right),-138.6$ $\left(\mathrm{s}, 1 \mathrm{~F}_{\infty \mathrm{eq}} ; J_{\mathrm{SiF}(\mathrm{eq})}=212.5 \mathrm{~Hz}\right)$. ${ }^{29 \mathrm{Si}} \mathrm{NMR}\left(\mathrm{CD}_{3} \mathrm{CN}\right.$ and $\left.\mathrm{CDCl}_{3}\right)$ : Experiments failed (under the same conditions as used successfully for $\left.7^{10}\right)$. EI MS: $m / z 165\left(10 \%,[\mathrm{M}-\mathrm{HF}]^{+}\right), 84\left(100 \%, \mathrm{CH}_{2}=\mathrm{NC}_{4} \mathrm{H}_{8}{ }^{+}\right)$. FAB MS (negative ions): $m / 2184\left(54 \%, M-H^{+}\right), 153$ (100\%, matrix). FAB MS (positive ions): $m / z 166\left(100 \%, M-F^{-}\right)$. Anal. Calcd for $\mathrm{C}_{6} \mathrm{H}_{14} \mathrm{~F}_{3} \mathrm{NSi}$ : C, 38.90; H, 7.62; F, 30.76; N, 7.56. Found: $\mathrm{C}, 39.1 ; \mathrm{H}$, 7.6; F, 30.8; N, 7.6.

Trifluoro(phenyl) (pyrrolidisiomethyl)silleate (10). A solution of 13 $(2.51 \mathrm{~g}, 9.98 \mathrm{mmol})$ in ethanol $(10 \mathrm{~mL})$ was added dropwise at $0^{\circ} \mathrm{C}$ during $5 \mathrm{~min}$ to a stirred solution of $40 \%$ hydrofluoric acid $(2.8 \mathrm{~g}, 56$ $\mathrm{mmol}$ of HF) in ethanol $(10 \mathrm{~mL})$. After stirring for $2 \mathrm{~h}$ at $0^{\circ} \mathrm{C}$, the precipitate was filtered off and recrystallized from methanol [cooling of a saturated $\left(20^{\circ} \mathrm{C}\right)$ solution to $-30^{\circ} \mathrm{C}$ ] to give $2.01 \mathrm{~g}$ (yield $\left.81 \%\right)$ of prismatic, colorless crystals; mp $125^{\circ} \mathrm{C}$. ${ }^{1} \mathrm{H}$ NMR $\left(\mathrm{CD}_{3} \mathrm{CN}\right): \delta 1.85-$ $2.05\left(\mathrm{~m}, 4 \mathrm{H}, \mathrm{CCH}_{2} \mathrm{C}\right), 2.56\left(\mathrm{~s}, 2 \mathrm{H}, \mathrm{SiCH}_{2} \mathrm{~N}\right), 2.85-3.0$ and 3.5-3.7 ( $\left.\mathrm{m}, 4 \mathrm{H}, \mathrm{NCH}_{2} \mathrm{C}\right), 6.9-7.9\left(\mathrm{~m}, 5 \mathrm{H}, \mathrm{SiC}_{6} \mathrm{H}_{5}\right), \mathrm{NH}$ resonance not resolved. ${ }^{13} \mathrm{C} N \mathrm{NR}\left(\mathrm{CD}_{3} \mathrm{CN}\right): 823.8\left(\mathrm{CCH}_{2} \mathrm{C}\right), 48.4\left(\mathrm{SiCH}_{2} \mathrm{~N}\right), 58.1\left(\mathrm{NCH}_{2} \mathrm{C}\right)$, $127.9\left(\mathrm{C}-3 / \mathrm{C}-5, \mathrm{SiC}_{6} \mathrm{H}_{5}\right), 129.2\left(\mathrm{C}-2 / \mathrm{C}-6, \mathrm{SiC}_{6} \mathrm{H}_{5}\right), 129.6\left(\mathrm{C}-4, \mathrm{SiC}_{6} \mathrm{H}_{5}\right)$, 137.9 (C.1, $\left.\mathrm{SiC}_{6} \mathrm{H}_{5}\right)$. ${ }^{19} \mathrm{~F}$ NMR $\left(\mathrm{CD}_{3} \mathrm{CN}\right): \delta-118.8$ (broad s). ${ }^{29} \mathrm{Si}$ NMR $\left(\mathrm{CD}_{3} \mathrm{CN}\right)$ : Experiments failed (under the same conditions as used successfully for $\left.7^{10}\right)$. EI MS: $m / z 227\left(26 \%,[M-H F]^{+}\right), 84(100 \%$, $\mathrm{CH}_{2}-\mathrm{NC}_{4} \mathrm{H}_{8}{ }^{+}$). Anal. Calcd for $\mathrm{C}_{11} \mathrm{H}_{16} \mathrm{~F}_{3} \mathrm{NSi}$ : C, 53.42; $\mathrm{H}, 6.52 ; \mathrm{F}$, 23.04; N, 5.66. Found: $C, 53.4 ; H, 6.6 ; F, 23.1 ; N, 5.7$.

(3-Aminopropyl)triethoxydilane (11) was obtained from WackerChemie GmbH, Burghausen, Germany.

Dimethoxy (methyl) (pyrrolidinomethyl)sllane (12). Synthesis as described in ref 5 .

Dimethoxy (phenyl)(pyrrolidinomethyl)ellane (13). A Grignard reagent was prepared from chlorobenzene $(13.5 \mathrm{~g}, 120 \mathrm{mmol})$ and magnesium turnings $(2.92 \mathrm{~g}, 120 \mathrm{mmol})$ in tetrahydrofuran $(100 \mathrm{~mL})$ and was then added dropwise at $0^{\circ} \mathrm{C}$ during $1 \mathrm{~b}$ to a stirred solution of $14(24.0 \mathrm{~g}, 117 \mathrm{mmol})$ in diethyl ether $(250 \mathrm{~mL})$. The mixture was stirred for $16 \mathrm{~h}$ at room temperature and then heated under reflux for $8 \mathrm{~h}$. The precipitate was filtered off and washed with $n$-pentane $(3 \times 50$ $\mathrm{mL})$, the filtrate was combined with the washings, and the solvent was removed by distillation at normal pressure. The residue was distilled in vacuo (Vigreux column) to give $15.9 \mathrm{~g}$ (yield 54\%) of a colorless liquid; bp $89^{\circ} \mathrm{C}$ (0.02 Torr). ' $\mathrm{H} N \mathrm{NR}\left(\mathrm{CDCl}_{3}\right)$ : $81.68-1.71\left(\mathrm{~m}, 4 \mathrm{H}, \mathrm{CCH}_{2} \mathrm{C}\right)$, $2.29\left(\mathrm{~s}, 2 \mathrm{H}, \mathrm{SiCH}_{2} \mathrm{~N}\right), 2.43-2.47\left(\mathrm{~m}, 4 \mathrm{H}, \mathrm{NCH}_{2} \mathrm{C}\right), 3.60(\mathrm{~s}, 6 \mathrm{H}$, $\left.\mathrm{SiOCH}_{3}\right), 7.34-7.41$ and $7.65-7.68\left(\mathrm{~m}, 5 \mathrm{H}, \mathrm{SiC}_{8} \mathrm{H}_{3}\right) .{ }^{13} \mathrm{C} \mathrm{NMR}$ 
Table I. Crystallographic Data for 9 and 10

\begin{tabular}{|c|c|c|}
\hline & 9 & 10 \\
\hline $\begin{array}{l}\text { chem formula } \\
\text { fw } \\
\text { space group } \\
T,{ }^{\circ} \mathrm{C} \\
a, \AA \\
b, \AA \\
c, \AA \\
\beta, d e g \\
V, A^{3} \\
Z\end{array}$ & $\begin{array}{l}\mathrm{C}_{6} \mathrm{H}_{14} \mathrm{~F}_{3} \mathrm{NSi} \\
185.3 \\
P 2_{1} / n \\
-100 \\
8.359(3) \\
11.388(4) \\
9.076(2) \\
93.40(3) \\
862.4(5) \\
4\end{array}$ & $\begin{array}{l}\mathrm{C}_{11} \mathrm{H}_{16} \mathrm{~F}_{3} \mathrm{NSi} \\
247.3 \\
P 2_{1} / n \\
-100 \\
9.542(4) \\
12.536(4) \\
10.996(3) \\
114.13(3) \\
1200.4(7) \\
4\end{array}$ \\
\hline $\begin{array}{l}\rho_{\text {alod, }} 8 \cdot \mathrm{cm}^{-3} \\
\mu(\mathrm{Mo} \mathrm{K \alpha}), \mathrm{cm}^{-1} \\
\text { no of unique reflns } \\
\text { no of reflns, used for refinement } \\
R^{a} \\
R_{w} \\
S, \text { goodness of fit }\end{array}$ & $\begin{array}{l}1.427 \\
2.53 \\
1521 \\
1182 \\
0.037 \\
0.037 \\
1.72\end{array}$ & $\begin{array}{l}1.369 \\
2.00 \\
2118 \\
1448 \\
0.043 \\
0.043 \\
1.36\end{array}$ \\
\hline
\end{tabular}

$\left(\mathrm{CDCl}_{3}\right): \& 23.9\left(\mathrm{CCH}_{2} \mathrm{C}\right), 42.6\left(\mathrm{SiCH}_{2} \mathrm{~N}\right), 50.8\left(\mathrm{SiOCH}_{3}\right), 58.0$ $\left(\mathrm{NCH}_{2} \mathrm{C}\right), 127.9\left(\mathrm{C}-3 / \mathrm{C}-5, \mathrm{SiC}_{6} \mathrm{H}_{5}\right), 130.2\left(\mathrm{C}-4, \mathrm{SiC}_{6} \mathrm{H}_{5}\right), 132.8(\mathrm{C}-1$ $\left.\mathrm{SiC}_{6} \mathrm{H}_{5}\right), 134.4\left(\mathrm{C}-2 / \mathrm{C}-6, \mathrm{SiC}_{6} \mathrm{H}_{5}\right)$. ${ }^{29 \mathrm{Si} \mathrm{NMR}}\left(\mathrm{CDCl}_{3}\right): \delta-20.6$. EI MS: $m / z 251\left(12 \%, \mathrm{M}^{+}\right), 84\left(100 \%, \mathrm{CH}_{2}=\mathrm{NC}_{4} \mathrm{H}_{3}^{+}\right)$. Anal. Calcd for $\mathrm{C}_{13} \mathrm{H}_{21} \mathrm{NO}_{2} \mathrm{Si} ; \mathrm{C}, 62.11 ; \mathrm{H}, 8.42 ; \mathrm{N}, 5.57$. Found $\mathrm{C}, 62.3 ; \mathrm{H}, 8.4 ; \mathrm{N}$, 5.5.

Trimethoxy(pyrrolldinomethyl)silane (14). Synthesis as described in ref 5 .

Methoxy(methyl)phenyl(pyrrolidinomethyl)silane (15). A Grignard reagent was prepared from bromobenzene $(1.57 \mathrm{~g}, 10.0 \mathrm{mmol})$ and magnesium turnings (243 mg, $10.0 \mathrm{mmol})$ in diethyl ether $(10 \mathrm{~mL})$ and was then added dropwise at $0^{\circ} \mathrm{C}$ during $20 \mathrm{~min}$ to a stirred solution of $12(1.89 \mathrm{~g}, 9.98 \mathrm{mmol})$ in diethyl ether $(50 \mathrm{~mL})$. The mixture was stirred for $20 \mathrm{~h}$ at room temperature and then heated under reflux for $2 \mathrm{~h}$. The precipitate was filtered off and washed with $n$-pentane $(50 \mathrm{~mL})$, the filtrate was combined with the washings, and the solvent was removed by distillation at normal pressure. The residue was distilled in vacuo (micro-Vigreux column) to give $1.19 \mathrm{~g}$ (yield $51 \%$ ) of a colorless liquid; bp $88^{\circ} \mathrm{C}$ (0.05 Torr). ' $\mathrm{H}$ NMR (CDCl $): \delta 0.05\left(\mathrm{~s}, 3 \mathrm{H}, \mathrm{SiCH}_{3}\right)$, 1.73-1.78 (m, $\left.4 \mathrm{H}, \mathrm{CCH}_{2} \mathrm{C}\right), 2.34\left(\mathrm{~s}, 2 \mathrm{H}, \mathrm{SiCH}_{2} \mathrm{~N}\right), 2.48-2.54(\mathrm{~m}, 4$ $\left.\mathrm{H}, \mathrm{NCH}_{2} \mathrm{C}\right), 3.51(\mathrm{~s}, 3 \mathrm{H}, \mathrm{SiOCH})_{3}, 7.36-7.42$ and $7.62-7.66(\mathrm{~m}, 5 \mathrm{H}$, $\left.\mathrm{SiC}_{6} \mathrm{H}_{5}\right)$. ${ }^{13} \mathrm{C} \mathrm{NMR}\left(\mathrm{CDCl}_{3}\right): \delta-3.5\left(\mathrm{SiCH}_{3}\right), 24.5\left(\mathrm{CCH}_{2} \mathrm{C}\right), 45.9$ $\left(\mathrm{SiCH}_{2} \mathrm{~N}\right), 51.4\left(\mathrm{SiOCH}_{3}\right), 58.6\left(\mathrm{NCH}_{2} \mathrm{C}\right), 128.6\left(\mathrm{C}-3 / \mathrm{C}-5, \mathrm{SiC}_{6} \mathrm{H}_{5}\right)$, $130.2\left(\mathrm{C}-4, \mathrm{SiC}_{6} \mathrm{H}_{5}\right), 134.2\left(\mathrm{C}-2 / \mathrm{C}-6, \mathrm{SiC}_{6} \mathrm{H}_{5}\right), 137.0\left(\mathrm{C}-1, \mathrm{SiC}_{6} \mathrm{H}_{5}\right)$. ${ }^{29} \mathrm{Si}$ NMR $\left(\mathrm{CDCl}_{3}\right): \delta 4.1$. EI MS: $m / z 235\left(19 \%, \mathrm{M}^{+}\right), 84(100 \%$, $\mathrm{CH}_{2}-\mathrm{NC}_{4} \mathrm{H}_{8}{ }^{+}$. Anal. Calcd for $\mathrm{C}_{13} \mathrm{H}_{21} \mathrm{NOSi}$ : C, 66.33; $\mathrm{H}, 8.99 ; \mathrm{N}$, 5.95. Found: $\mathrm{C}, 66.4 ; \mathrm{H}, 8.9 ; \mathrm{N}, 6.0$.

(b) X-ray Diffraction Studles, Cell parameters were obtained from least-squares fits to the settings of 25 reflections in the range $15^{\circ} \leq \theta$ $\leq 20^{\circ}$ centered on a Siemens P4 diffractometer by using Mo Ka radiation. Intensities were collected for colorless, prismatic crystals of 9 and 10 at $-100{ }^{\circ} \mathrm{C}$ for $2 \theta \leq 50^{\circ}$. No significant deviations in intensity were registered for three monitor reflections recorded at regular intervals. Crystallographic parameters and additional details of the data collection and refinement are given in Table I. A total of $1521\left(R_{\mathrm{Lt}}=0.015\right)$ and $2118\left(R_{\text {int }}=0.029\right)$ independent reflections were measured for 9 and 10 , respectively. On the basis of the rejection criterion $F_{0}{ }^{2} \leq 2 \sigma\left(F_{0}{ }^{2}\right) 1182$ and 1448 reflections were used for subsequent refinements. The structures were solved by direct methods and refined by full-matrix least-squares ${ }^{13}$ with anisotropic thermal parameters for all non-hydrogen atoms. Neutralatom scattering factors, corrected for the real and imaginary parts of anomalous dispersion, were taken from ref 14 . Corrections for absorption were applied after isotropic least-squares refinement for the non-bydrogen atoms by use of the program DIFABS. ${ }^{15}$ All the hydrogen atoms were located in difference Fourier syntheses and their positions included in the least-squares refinement together with isotropic temperature factors. The function minimized during the refinement was $\Sigma w\left(\left|F_{d}-\right| F_{d}\right)^{2}$ with $w=$ $\left[\sigma^{2}\left(F_{0}\right)+p F_{0}^{2}\right]^{-1}, p=0.0001$ for 9 and 0.0003 for 10 . Atom coordinates

(13) Sheldrick, G. M. SHELX-76. University of Cambridge, Cambridge, England, 1976. SHELXTL PLUS. Siemens Analytical X-Ray Instruments Inc., 1990.

(14) International Tables for X-Ray Crystallography; Kynoch Press: Birmingham, England, 1974; Vol. IV, pp 99-149.

(15) Walker, N.; Stuart, D. Acta Crysiallogr. 1983, A39, 158-166.
Table II. Atom Coordinates $\left(\times 10^{4}\right)$ and Temperature Factors $\left(\boldsymbol{A}^{2}\right.$ $\left.\times 10^{3}\right)$ for 9

\begin{tabular}{lrrrr}
\hline atom & \multicolumn{1}{c}{$x$} & \multicolumn{1}{c}{$y$} & $z$ & $U($ eq) \\
\hline $\mathrm{Si}$ & $2003(1)$ & $143(1)$ & $2924(1)$ & $23(1)$ \\
$\mathrm{F}(1)$ & $91(2)$ & $472(1)$ & $3460(1)$ & $26(1)$ \\
$\mathrm{F}(2)$ & $3872(2)$ & $-294(1)$ & $2571(2)$ & $37(1)$ \\
$\mathrm{F}(3)$ & $1435(2)$ & $-1226(1)$ & $2898(2)$ & $40(1)$ \\
$\mathrm{C}(1)$ & $1709(4)$ & $1031(3)$ & $1217(3)$ & $34(1)$ \\
$\mathrm{C}(2)$ & $2914(3)$ & $774(2)$ & $4720(3)$ & $26(1)$ \\
$\mathrm{C}(3)$ & $2616(4)$ & $1706(3)$ & $7204(3)$ & $31(1)$ \\
$\mathrm{C}(4)$ & $1837(4)$ & $2833(3)$ & $7648(3)$ & $34(1)$ \\
$\mathrm{C}(5)$ & $1688(4)$ & $3511(3)$ & $6219(3)$ & $39(1)$ \\
$\mathrm{C}(6)$ & $1246(4)$ & $2603(2)$ & $5058(3)$ & $35(1)$ \\
$\mathrm{N}$ & $1846(2)$ & $1440(2)$ & $5692(2)$ & $21(1)$
\end{tabular}

a The equivalent isotropic temperature factor $U(e q)$ is defined as onethird of the trace of the orthogonalized $U_{i j}$ tensor.

Table III. Atom Coordinates $\left(\times 10^{4}\right)$ and Temperature Factors $\left(A^{2}\right.$ $\left.\times 10^{3}\right)$ for 10

\begin{tabular}{lrrrr}
\hline atom & \multicolumn{1}{c}{$\boldsymbol{x}$} & \multicolumn{1}{c}{$y$} & $\boldsymbol{z}$ & $U(\mathrm{eq})^{\boldsymbol{e}}$ \\
\hline $\mathrm{Si}$ & $630(1)$ & $3295(1)$ & $4221(1)$ & $34(1)$ \\
$\mathrm{F}(1)$ & $500(2)$ & $4645(1)$ & $3944(1)$ & $33(1)$ \\
$\mathrm{F}(2)$ & $672(2)$ & $1991(1)$ & $4593(2)$ & $61(1)$ \\
$\mathrm{F}(3)$ & $-1225(2)$ & $3298(1)$ & $3780(2)$ & $47(1)$ \\
$\mathrm{C}(1)$ & $1303(3)$ & $3051(2)$ & $2869(3)$ & $37(1)$ \\
$\mathrm{C}(2)$ & $1888(4)$ & $3515(3)$ & $6052(3)$ & $39(1)$ \\
$\mathrm{C}(3)$ & $3337(4)$ & $4794(3)$ & $7943(3)$ & $46(1)$ \\
$\mathrm{C}(4)$ & $4764(5)$ & $5432(3)$ & $8224(4)$ & $58(2)$ \\
$\mathrm{C}(5)$ & $4852(4)$ & $5626(3)$ & $6909(3)$ & $56(2)$ \\
$\mathrm{C}(6)$ & $3891(4)$ & $4764(3)$ & $6001(3)$ & $48(2)$ \\
$\mathrm{C}(12)$ & $2250(4)$ & $2191(3)$ & $2902(4)$ & $56(2)$ \\
$\mathrm{C}(13)$ & $2727(5)$ & $2004(4)$ & $1879(6)$ & $76(2)$ \\
$\mathrm{C}(14)$ & $2241(5)$ & $2665(5)$ & $795(5)$ & $81(2)$ \\
$\mathrm{C}(15)$ & $1293(5)$ & $3507(4)$ & $716(4)$ & $72(2)$ \\
$\mathrm{C}(16)$ & $844(4)$ & $3697(3)$ & $1742(3)$ & $50(1)$ \\
$\mathrm{N}$ & $2631(3)$ & $4577(2)$ & $6455(2)$ & $32(1)$
\end{tabular}

- The equivalent isotropic temperature factor $U(e q)$ is defined as onethird of the trace of the orthogonalized $U_{i j}$ tensor.

Table IV. Selected Bond Distances ( $\AA$ ) and Angles (deg) for 9 and 10

\begin{tabular}{lll} 
& 9 & 10 \\
\hline Si-F(1) & $1.738(2)$ & $1.715(2)$ \\
Si-F(2) & $1.689(2)$ & $1.681(2)$ \\
Si-F(3) & $1.630(2)$ & $1.633(2)$ \\
Si-C(1) & $1.854(3)$ & $1.870(4)$ \\
Si-C(2) & $1.899(3)$ & $1.896(3)$ \\
C(2)-N & $1.498(3)$ & $1.487(4)$ \\
C(3)-N & $1.512(3)$ & $1.518(4)$ \\
C(6)-N & $1.513(3)$ & $1.498(5)$ \\
C(3)-C(4) & $1.504(4)$ & $1.498(6)$ \\
C(4)-C(5) & $1.509(4)$ & $1.503(6)$ \\
C(5)-C(6) & $1.500(4)$ & $1.502(5)$ \\
F(1)-Si-F(2) & $173.1(1)$ & $174.5(1)$ \\
F(1)-Si-F(3) & $86.5(1)$ & $87.4(1)$ \\
F(1)-Si-C(1) & $92.2(1)$ & $92.5(1)$ \\
F(1)-Si-C(2) & $90.4(1)$ & $90.8(1)$ \\
F(2)-Si-F(3) & $89.3(1)$ & $89.4(1)$ \\
F(2)-Si-C(1) & $94.6(1)$ & $93.0(1)$ \\
F(2)-Si-C(2) & $86.9(1)$ & $86.7(1)$ \\
F(3)-Si-C(1) & $119.1(1)$ & $116.5(1)$ \\
F(3)-Si-C(2) & $118.4(1)$ & $117.0(2)$ \\
C(1)-Si-C(2) & $122.5(1)$ & $126.4(2)$ \\
Si-C(2)-N & $118.3(2)$ & $117.7(2)$ \\
& &
\end{tabular}

are listed in Tables II and III, selected bond lengths and angles in Table IV. The atomic numbering schemes are given in Figures 1 and 2.

(c) Solld-State NMR Studies. High-resolution solid-state ${ }^{15} \mathrm{~N}$ and 29Si NMR spectra of 7 and 8 have been obtained on a Bruker MSL-300 NMR spectrometer operating at $30.4\left({ }^{15} \mathrm{~N}\right)$ and $59.6\left({ }^{29} \mathrm{Si}\right) \mathrm{MHz}$, respectively. Chemical shifts are given with respect to external TMS ( 0 ppm, ${ }^{29} \mathrm{Si}$ ) and solid $\mathrm{NH}_{4} \mathrm{NO}_{3}\left(\mathrm{NO}_{3}-\right.$ resonance, $\left.0 \mathrm{ppm},{ }^{15} \mathrm{~N}\right)$. The Hartmann-Hahn matching condition for ${ }^{1} \mathrm{H} \rightarrow{ }^{15} \mathrm{~N}$ experiments was set on slow-spinning solid $\mathrm{NH}_{4} \mathrm{NO}_{3}$, using a $5-\mu \mathrm{s}{ }^{1} \mathrm{H} 90^{\circ}$ pulse. The experimental parameters for the ${ }^{1} \mathrm{H} \rightarrow{ }^{15} \mathrm{~N}$ CP MAS spectra of 7 and 


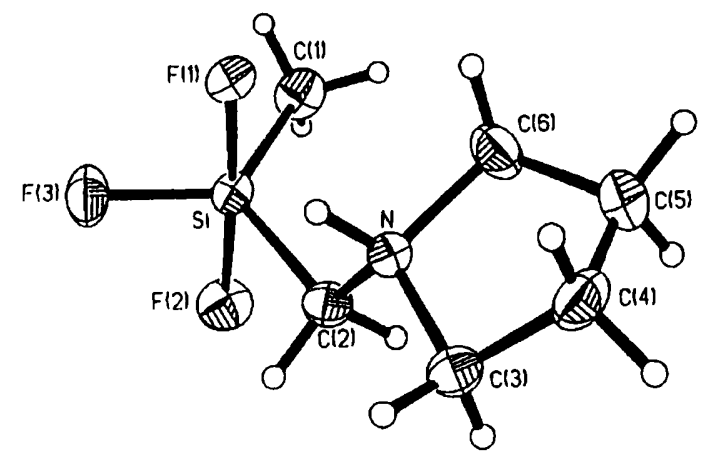

Figure 1. Molecular structure of 9 (ORTEP plot) in the crystal, showing the atomic numbering scheme. The thermal ellipsoids are drawn at a $50 \%$ probability level.

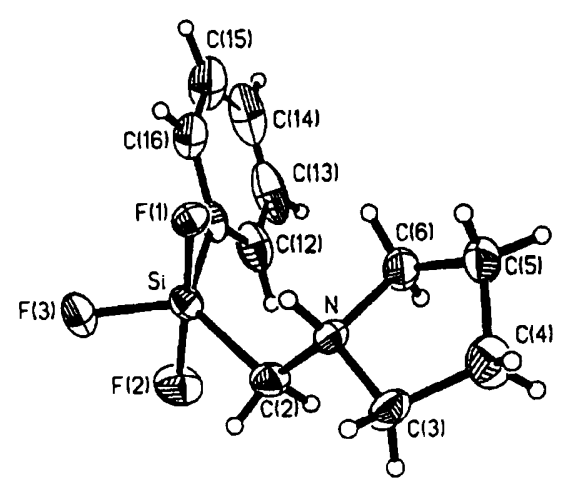

Figure 2. Molecular structure of 10 (ORTEP plot) in the crystal, showing the atomic numbering scheme. The thermal ellipsoids are drawn at a $50 \%$ probability level.

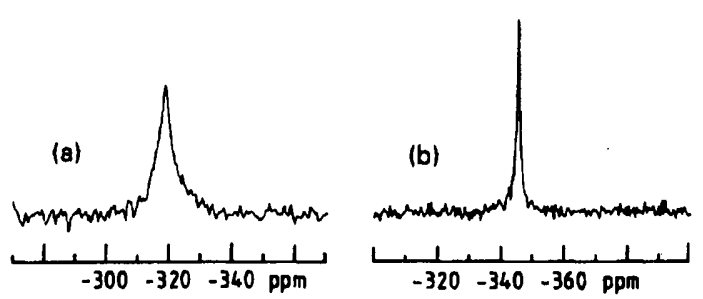

Figure 3. ${ }^{1} \mathrm{H} \rightarrow{ }^{15} \mathrm{~N}$ CP MAS spectra of (a) 7 and (b) 8. Key: (a) spinning rate $=2.9 \mathrm{kHz}$, contact time $=8 \mathrm{~ms}$, recycle delay time $=20$ s, 3188 transients, no exponential line broadening, $8\left({ }^{15} \mathrm{~N}\right)=-318.5 \mathrm{ppm}$, $\nu_{1 / 2}=140 \mathrm{~Hz}$; (b) spinning rate $=3.0 \mathrm{kHz}$, contact time $=5 \mathrm{~ms}$, recycle delay time $=8 \mathrm{~s}, 922$ transients, no exponential line broadening, $\delta\left({ }^{15} \mathrm{~N}\right)$ $=-345.3 \mathrm{ppm}, \nu_{1 / 2}=35 \mathrm{~Hz}$.

8 are given in the respective figure caption (Figure 3). The procedure for setting the Hartmann-Hahn matching condition for ${ }^{19} \mathrm{~F} \rightarrow{ }^{29} \mathrm{Si} \mathrm{CP}$ MAS experiments (using a $5-\mu s^{19} \mathrm{~F} 90^{\circ}$ pulse) on topaz, $\left[\mathrm{Al}_{2}(\mathrm{~F}, \mathrm{OH})_{2}\right.$ (SiO) ] (with >95\% fluorine content), as well as the necessary spectrometer hardware modifications, has been described elsewhere. ${ }^{16}$ Again, experimental parameters of the ${ }^{19} \mathrm{~F} \rightarrow{ }^{29} \mathrm{Si} \mathrm{CP}$ MAS and of the singlepulse ${ }^{29} \mathrm{Si}$ MAS spectra with high-power ${ }^{19} \mathrm{~F}$-decoupling are given in the respective figure caption (Figure 4), ${ }^{1} \mathrm{H} \rightarrow{ }^{29} \mathrm{Si} \mathrm{CP}$ MAS experiments were set up using $\mathrm{Q}_{8} \mathrm{M}_{8}$ and a $5-\mu \mathrm{s}^{1} \mathrm{H} 90^{\circ}$ pulse.

\section{Results and Discussion}

(a) Syntheses. Tetrafluoro(pyrrolidiniomethyl)silicate ${ }^{10}$ (7) was synthesized as described in the literature. (3-Ammoniopropyl)tetrafluorosilicate (8) was prepared analogously by reaction of commercially available (3-aminopropyl)triethoxysilane (11) with an excess of hydrogen fluoride in ethanol/hydrofluoric acid at $0^{\circ} \mathrm{C}$, following the strategy outlined in ref 12 , but using a modified procedure (Scheme III). 8 was isolated with $80 \%$

(16) Sobald, A.; Merwin, L. H.; Schaller, T.; Knoller, W. J. Magn. Reson. 1992, 96, 159-164.
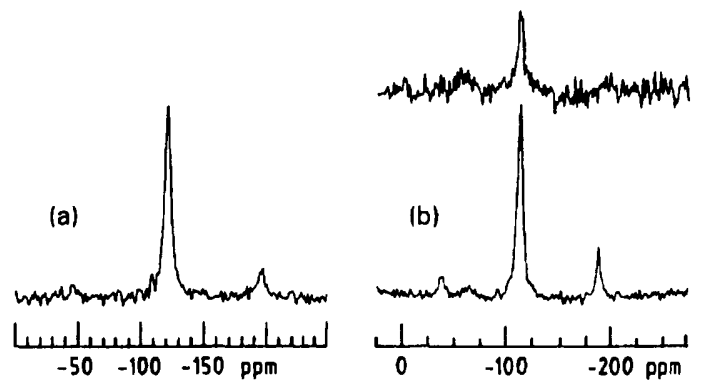

Figure 4. ${ }^{29} \mathrm{Si}$ MAS spectrum of (a) 7 as well as ${ }^{29} \mathrm{Si}$ MAS and ${ }^{19} \mathrm{~F} \rightarrow$ ${ }^{29} \mathrm{Si}$ CP MAS spectra of (b) 8. Key: (a) ${ }^{19} \mathrm{~F}$ high-power docoupled ${ }^{29} \mathrm{Si}$ MAS spectrum of 7, spinning rate $=4.5 \mathrm{kHz}, 30^{\circ} 29 \mathrm{Si}$ pulse, recycle delay time $=308,2271$ transients, exponential line broadening of $25 \mathrm{~Hz}$, $\delta\left({ }^{29} \mathrm{Si}\right)=-121.1 \mathrm{ppm}, \nu_{1 / 2}=350 \mathrm{~Hz}$; (b) ${ }^{19} \mathrm{~F} \rightarrow 29 \mathrm{Si} \mathrm{CP}$ MAS spectrum of 8 (top), with spinning rate $=4.5 \mathrm{kHz}$, contact time $-10 \mathrm{~ms}$, recycle delay time $=15 \mathrm{~s}, 1167$ transients, exponential line broadening of $25 \mathrm{~Hz}$, and ${ }^{19} \mathrm{~F}$ high-power decoupled ${ }^{29} \mathrm{Si}$ MAS spectrum of 8 (bottom) with spinning rate $=4.5 \mathrm{kHz}, 30^{\circ} \% \mathrm{Si}$ pulse, recycle delay time $=30 \mathrm{~s}, 2165$ transients, exponential line broadening of $25 \mathrm{~Hz}, 8\left({ }^{29} \mathrm{Si}\right)=-112.4 \mathrm{ppm}$, $v_{1 / 2}=300 \mathrm{~Hz}$

Scheme III

$$
\begin{aligned}
& \begin{array}{l}
\mathrm{OC}_{2} \mathrm{H}_{5} \\
\mathrm{C}_{2} \mathrm{H}_{5} \mathrm{O}-\mathrm{Si}_{1} \mathrm{CH}_{2}-\mathrm{CH}_{2}-\mathrm{CH}_{2}-\mathrm{NH}_{2} \\
1 \\
\mathrm{OC}_{2} \mathrm{H}_{5} \quad 11
\end{array} \\
& \text { + } 4 \mathrm{HF} \\
& \text { - } 3 \mathrm{C}_{2} \mathrm{H}_{5} \mathrm{OH}
\end{aligned}
$$

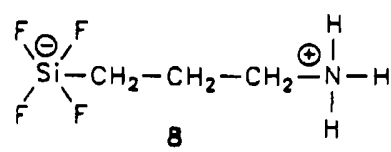

Scheme IV

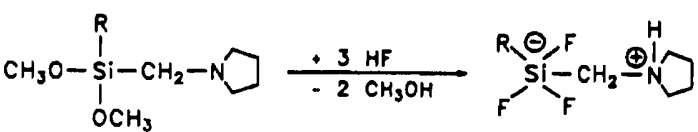

$$
\begin{aligned}
& \begin{array}{r|l} 
& R \\
\hline 0,12 & \mathrm{CH}_{3} \\
10,13 & \mathrm{C}_{3} \mathrm{H}_{5}
\end{array}
\end{aligned}
$$<smiles>CO[Si](C)(CN1CCCC1)OC</smiles>

14<smiles>CO[Si](CN1CCCC1)(OC)c1ccccc1</smiles>

13 yield (lit. ${ }^{12 \pi} 50 \%$ ) as a colorless crystalline solid. Similarly, trifluoro(methyl)(pyrrolidiniomethyl)silicate (9) and trifluoro(phenyl)(pyrrolidiniomethyl)silicate (10) were synthesized by reaction of dimethoxy (methyl)(pyrrolidinomethyl)silane (12) and dimethoxy (phenyl)(pyrrolidinomethyl)silane (13), respectively, with an excess of hydrogen fluoride in ethanol/hydrofluoric acid at $0^{\circ} \mathrm{C}$ (Scheme IV). The new zwitterionic organofluorosilicates were isolated with $90 \%$ (9) and $81 \%$ (10) yield as colorless crystalline solids. 9 and 10 are the first representatives of the hitherto unknown class of zwitterionic (ammonioalkyl)organotrifluorosilicates. 
Scheme V

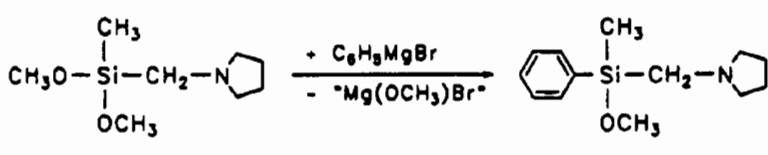

12

$$
\begin{aligned}
& 15
\end{aligned}
$$

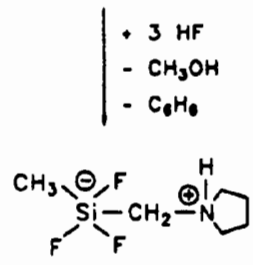

9

Alternatively, 9 was obtained by reaction of methoxy (methyl)phenyl (pyrrolidinomethyl)silane (15) with an excess of hydrogen fluoride in ethanol/hydrofluoric acid at $0^{\circ} \mathrm{C}$ (yield 89\%; Scheme V). In addition to the $\mathrm{Si}-\mathrm{O}$ bond cleavage (generation of methanol), the formation of 9 from 15 involves a chemoselective $\mathrm{Si}-\mathrm{C}$ bond cleavage (generation of benzene). No indications were found for the formation of methane ( $\mathrm{Si}-\mathrm{CH}_{3}$ cleavage) or 1-methylpyrrolidine ( $\mathrm{Si}-\mathrm{CH}_{2} \mathrm{NC}_{4} \mathrm{H}_{8}$ cleavage). In this context it should be mentioned that the zwitterionic organofluorosilicate 10 was found to decompose almost completely via a Si- $\mathrm{C}_{6} \mathrm{H}_{3}$ cleavage (formation of benzene) upon heating in $\mathrm{CDCl}_{3}$ at $50^{\circ} \mathrm{C}$ for 5 hours ( ${ }^{1} \mathrm{H}$ and ${ }^{13} \mathrm{C} \mathrm{NMR}$ ).

The starting materials used for the synthesis of the zwitterionic silicates 9 and 10 were prepared according to the literature $\left(12,{ }^{3}\right.$ $\left.14^{5}\right)$ or were synthesized for the first time $(13$, Scheme IV; 15 , Scheme V). Details of the preparation and characterization of the new silanes 13 and 15 are given in the Experimental Section.

The identity of 8 was proven by elemental analyses $(C, H, F$, $\mathrm{N})$, by mass-spectrometric investigations (EIMS), and by solution and solid-state NMR studies (solution in $\mathrm{CD}_{3} \mathrm{OD},{ }^{1} \mathrm{H}$ and ${ }^{13} \mathrm{C}$ NMR; solid state, ${ }^{15} \mathrm{~N}$ and ${ }^{29} \mathrm{Si} \mathrm{NMR}$ ). These investigations (see the Experimental Section and Solid-State NMR Data) unequivocally establish the zwitterionic structure of 8 as was postulated in ref 12 .

The identity of 9 and 10 was established by elemental analyses (C, H, F, N), mass-spectrometric investigations (9, EI MS, FAB MS; 10, EI MS) and solution-state NMR studies (see Experimental Section). In addition, the crystal structures of 9 and 10 were established by X-ray diffraction (see Crystal Structures).

The zwitterionic nature of 9 and $\mathbf{1 0}$ is reflected by their low solubility in organic solvents. For solutions of $9\left(\mathrm{CD}_{3} \mathrm{CN}, \mathrm{CDCl}_{3}\right)$ and $10\left(\mathrm{CD}_{3} \mathrm{CN}\right)$ similar ${ }^{1} \mathrm{H}$ and ${ }^{13} \mathrm{C}$ NMR spectra for the respective pyrrolidiniomethyl groups were obtained (see Experimental Section). The chemical shifts for the $\mathrm{NCH}_{2} \mathrm{C}$ protons of the pyrrolidinio moiety (multiplets at $2.8-3.0$ and $3.5-3.8 \mathrm{ppm}$ ) are typical of methylene groups bound to an ammonium-type nitrogen. This interpretation is supported by the ${ }^{1} \mathrm{H}$ NMR spectrum of 9 in $\mathrm{CDCl}_{3}$, showing a resonance signal at $\delta=8.0$, which is typical of an NH function of an ammonium group. In contrast to the related zwitterionic organofluorosilicate 7 [ $\delta$ $\left({ }^{29} \mathrm{Si}\right)=-120.8$; solution in $\left.\mathrm{CD}_{3} \mathrm{CN}\right],{ }^{10}$ all attempts to detect a 29Si resonance at room temperature for solutions of $9\left(\mathrm{CD}_{3} \mathrm{CN}\right.$, $\left.\mathrm{CDCl}_{3}\right)$ and $10\left(\mathrm{CD}_{3} \mathrm{CN}\right)$ failed. ${ }^{17}$ Thus, pentacoordination of the silicon atoms in solution could not be established directly. However, as the ${ }^{1} \mathrm{H}$ and ${ }^{13} \mathrm{CNMR}$ data for the pyrrolidiniomethyl group of 9 and 10 are very similar to those observed for 7 (whose pentacoordination was proved directly), it is concluded that the

(17) The failure to obeerve 2 Si resonances at room temperature for solutions of 9 and 10 may have to be attributed to motional processes involving the $\mathrm{SiF}_{3}$ moiety as has also been diecussed for related ionic compounds (see for example refs $1 c, e, f$ and $2 i, m, n, t)$. zwitterions 9 and 10 also exist in solution. This assumption is strongly supported by low-temperature solution-state ${ }^{19} \mathrm{~F}$ NMR studies of 9 in $\mathrm{CDCl}_{3}$. At $20^{\circ} \mathrm{C}$ a single broad ${ }^{19} \mathrm{~F}$-resonance signal, centered at $-106.0 \mathrm{ppm}\left(\nu_{1 / 2} \approx 1995 \mathrm{~Hz}\right)$, was observed, indicating a rapid exchange of the four fluorineatoms. On cooling, however, this ligand exchange becomes significantly slower, and at $-70^{\circ} \mathrm{C}$ two sharp ${ }^{19} \mathrm{~F}$-resonance signals at $\delta=-91.5$ (relative intensity $=2 ; J_{\mathrm{SiF}(\mathrm{xx})}=242.9 \mathrm{~Hz}$ ) and $\delta=-138.6$ (relative intensity $=1 ; J_{\mathrm{SiF}(\mathrm{co})}=212.5 \mathrm{~Hz}$ ) as well as ${ }^{19} \mathrm{~F} /{ }^{29} \mathrm{Si}$ spin-spin coupling were observed. These data are consistent with a trigonalbipyramidal geometry of 9 in solution, with two fluorine atoms in the axial sites and one fluorine atom in an equatorial position. This is in accordance with the geometries observed for 9 and $\mathbf{1 0}$ in the crystal.

(b) Crystal Structures. The molecular structures of the zwitterionic fluorosilicates 9 and 10 in the crystal are depicted in Figures 1 and 2. The fluorine atoms $F(1)$ and $F(2)$ occupy the axial sites in a distorted trigonal bipyramid (TBP), $F(3)$ and the carbon atoms $C(1)$ and $C(2)$ the equatorial positions. As may be gathered from Table IV, both molecules display similar deviations from an idealized TBP. The observed distortions are not in accordance with angular movements on the Berry pseudorotation pathway toward a square-pyramidal geometry.

The equatorial bond angles in 9 reflect the greater steric requirements of the bulkier pyrrolidiniomethyl and methyl group in comparison to the fluorine atom $F(3)$. As a consequence, the $\mathrm{C}(1)-\mathrm{Si}-\mathrm{C}(2)$ angle is $122.5(1)^{\circ}$ accompanied by a concomitant narrowing of the $F(3)-S i-C(1)$ and $F(3)-S i-C(2)$ angles to 119.1(1) and $118.4(1)^{\circ}$, respectively. Increased steric demands of the phenyl group leads to more pronounced deviations from the idealized equatorial angles in 10 . The $C(1)-S i-C(2)$ angle displays a value of $126.4(2)^{\circ}$; the $F(3)-S i-C(1)$ and $F(3)-S i-$ $C(2)$ angles are $116.5(1)$ and $117.0(2)^{\circ}$. The axial bond angles $\mathrm{F}(1)-\mathrm{Si}-\mathrm{F}(2)$ also deviate from the idealized value of $180^{\circ}[173.1$. (1) and $174.5(1)^{\circ}$, respectively]. The deformation parameter $\Delta,{ }^{2 j}$ defined as the sum of the axial and equatorial bond angle displacements from the ideal values of 180 and $120^{\circ}$, is 9.4 and $11.9^{\circ}$ in 9 and 10, respectively [angles used for these calculations: $F(1)-S i-F(2), C(1)-S i-C(2)]$. Similar values have been reported for ionic diorganotrifluorosilicates, for instance 8.7,11.4, and $12.4^{\circ}$ in the anions $\mathrm{Ph}_{2} \mathrm{SiF}_{3}-, 2 f, 2 \mathrm{~K} \mathrm{Mes}_{2} \mathrm{SiF}_{3}-, 20$ and $t$-BuPhSiF ${ }_{3}, 2 n$ respectively. The deviations of the $\mathrm{F}_{\mathrm{ax}}-\mathrm{Si}_{\mathrm{B}}-\mathrm{F}_{\mathrm{ma}}$ and $\mathrm{F}_{\mathrm{u}}-\mathrm{Si}-\mathrm{C}_{\mathrm{eq}}$ angles from the ideal value of $90^{\circ}$ are clearly a result of the steric requirements of the bulky equatorial groups. Whereas both the $F(1)-S i-C(1)$ and $F(2)-S i-C(1)$ angles in 9 and 10 are significantly wider than $90^{\circ}$ [range 92.2(1)-94.6$\left.(1)^{\circ}\right]$, the former axial fluorine atom is displaced toward $F(3)$, with the latter displaced toward $C(2)$.

Inspection of Figures 1 and 2 indicates that the pyrrolidiniomethyl groups in 9 and 10 both adopt conformations, which lead to short intramolecular $F(1) \cdots \mathrm{N}$ contacts. The torsion angles $F(1)-S i-C(2)-N$ in 9 and 10 are $-10.0(2)$ and $-17.4(3)^{\circ}$, respectively, the $F(1) \cdots N$ distances 2.67 and $2.68 \AA$. However, the intramolecular $(\mathrm{N}) \mathrm{H} \ldots \mathrm{F}(1)$ distances of 2.25 and $2.19 \AA$ are not indicative of a significant degree of hydrogen bonding; 9 and 10 display $\mathrm{Si}-\mathrm{C}(2)-\mathrm{N}-\mathrm{H}$ torsion angles of 51 and $48^{\circ}$, respectively. Relatively weak intermolecular $\mathrm{N}-\mathrm{H}$... $\mathrm{F}(1)$ hydrogen bonds $[F(1) \cdots N$ distance $2.85 \AA]$ lead to the formation of centrosymmetric dimers in the case of 9 . The intermolecular $(\mathrm{N}) \mathrm{H} \cdots \mathrm{F}(1)$ distance is $1.96 \mathrm{~A}$; the $\mathrm{N}-\mathrm{H} \cdots \mathrm{F}(1)$ angle is $152^{\circ}$. For 10 intermolecular $N . . . F(1)$ and $N$...F(3) distances of 3.00 and $2.95 \AA$ accompanied by $(\mathrm{N}) \mathrm{H} \ldots \mathrm{F}(1)$ and $(\mathrm{N}) \mathrm{H} \ldots \mathrm{F}(3)$ distances of 2.26 and $2.14 A$ are observed for analogous molecule pairs related by a crystallographic center of symmetry. Both pyrrolidinio ring systems display an envelope conformation with $\mathrm{C}(4)$ displaced by $0.59 \AA$ from the plane of the remaining atoms in 9. In contrast, the atoms $C(3), C(4), C(5)$, and $N$ are effectively coplanar in 10, with $\mathrm{C}(6)$ displaced by $0.50 \AA$ from this plane. 
The equatorial Si-F(3) bond lengths of $1.630(2)$ and 1.633(2) $A$ in the zwitterions 9 and 10 are similar to those observed for ionic diorganotrifluorosilicates. $2 n, 24$ The axial Si-F(1) bond is significantly longer than the opposite $\mathrm{Si}-\mathrm{F}(2)$ bond in both 9 (difference $0.049 \AA$ ) and 10 (difference $0.034 \AA$ ). A similar phenomenon has been reported for ionic diorganotrifluorosilicates with 18-crown-6 potassium as the cation. ${ }^{2 n, 25}$ In each case the axial fluorine atom of the longer $\mathrm{Si}-\mathrm{F}_{\mathrm{ax}}$ bond participates in the coordination sphere of the potassium cation and the relevant $F_{a x}-$ $\mathrm{Si}-\mathrm{F}_{\text {eq }}$ angle is markedly smaller than $90^{\circ}$, as is observed in 9 and 10. Different axial Si-F bond distances were also found for the zwitterionic organotetrafluorosilicate 7 (differences 0.045 and $0.049 \AA$, respectively), ${ }^{10}$ which may be explained by dipolar $F \cdots N$ interactions. The lengthening of the $\mathrm{Si}-\mathrm{F}(1)$ with respect to the $\mathrm{Si}-\mathrm{F}(2)$ distances in the zwitterionic silicates 9 and 10 may also be a result of dipolar $F(1) \ldots N$ interactions displayed by the former axial fluorine atom. In the case of 9 , intermolecular $\mathrm{N}-\mathrm{H} . . . \mathrm{F}$ hydrogen bonds may also play a role.

A dihedral angle of $57.8^{\circ}$ is observed in 10 between the plane of the phenyl ring and the equatorial plane of the TBP. The corresponding dihedral angle in $\left[\mathrm{N}(n-\mathrm{Bu})_{4}\right]\left[\mathrm{PhMeSiF}_{3}\right]$ is $78.2^{\circ},{ }^{2 \mathrm{j}}$

(c) Solid-State NMR Data. The zwitterionic organofluorosilicates 7-10 were studied by solid-state NMR spectroscopy (7, $\left.8,{ }^{15} \mathrm{~N} ; 7-10,{ }^{29} \mathrm{Si}\right) .{ }^{15} \mathrm{~N}$ CP MAS and ${ }^{29} \mathrm{Si}$ MAS and CP MAS spectra of 7 and 8 are shown in Figures 3 and 4.

The ${ }^{15} \mathrm{~N}$ chemical shifts for $7(-318.5 \mathrm{ppm})$ and $8(-345.3$ ppm) clearly characterize these ${ }^{15} \mathrm{~N}$ resonances as due to ammonium-type nitrogen. ${ }^{18}$ The larger line width of the ${ }^{15} \mathrm{~N}$ resonance of $7\left(\nu_{1 / 2}=140 \mathrm{~Hz}\right)$ as compared to $8\left(\nu_{1 / 2}=35 \mathrm{~Hz}\right)$ may have to be attributed to some residual ${ }^{19} \mathrm{~F}-1{ }^{15} \mathrm{~N}$ dipolar interactions in 7; the average ${ }^{19} \mathrm{~F} . . .15 \mathrm{~N}$ distances in 7 should be shorter than those in 8 . An alternative explanation can be found on the basis of the X-ray crystallographic data for 7.10 First, there are two crystallographically independent molecules per asymmetric unit, information which is apparently not resolved in the ${ }^{1} \mathrm{H} \rightarrow{ }^{15} \mathrm{~N}$ CP MAS spectrum of 7 (neither are the two expected ${ }^{29} \mathrm{Si}$ resonances resolved in the ${ }^{29} \mathrm{Si}$ MAS spectrum of 7, see Figure 4). Thus, the presence of two unresolved ${ }^{15} \mathrm{~N}$ resonances may account for some increase in the observed line width. Second and more important, some dynamic disorder phenomena (along with a phase transition occurring near -10 ${ }^{\circ} \mathrm{C}$ ) has been claimed for 7 on the basis of variable-temperature $\mathrm{X}$-ray diffraction studies. ${ }^{10}$ This latter crystallographic finding is in fact corroborated by the occurrence of a broadened ${ }^{15} \mathrm{~N}$ resonance. Another NMR-spectroscopic result further confirms this interpretation: while there is no problem to obtain a ${ }^{29} \mathrm{Si}$ single-pulse MAS spectrum of 7 (under ${ }^{19} \mathrm{~F}$ high-power decoupling conditions, see Figure 4), all our attempts to obtain a ${ }^{19} \mathrm{~F} \rightarrow{ }^{29} \mathrm{Si}$ CP MAS spectrum of 7 failed. A wide range of ${ }^{19} \mathrm{~F} \rightarrow{ }^{29} \mathrm{Si}$ cross polarization contact times (ranging from 1 to $25 \mathrm{~ms}$ ) have been used unsuccessfully. Taking the spin dynamics of the CP MAS experiment into account, motional processes, like the mentioned dynamic disorder phenomena for 7 or intramolecular exchange processes involving the $\mathrm{SiF}_{4}$ moiety, can partially (or fully) average the respective dipolar interactions, thus rendering the cross polarization process fairly (or totally) inefficient. Under such circumstances also MAS rates of only a few kilohertz may interfer with the CP process, leading to well-known modulations in the $\mathrm{CP}$ matching curves ${ }^{19}$ and to the potential complete failure of $\mathrm{CP}$ MAS methods for such cases. Further low-temperature CPMAS studies should enable us to prove this interpretation for compounds 7 and 8 .

Along with the ${ }^{29} \mathrm{Si}$ MAS spectrum of 7, Figure 4 also depicts the ${ }^{29} \mathrm{Si}$ MAS and ${ }^{19} \mathrm{~F} \rightarrow{ }^{29} \mathrm{Si} \mathrm{CP}$ MAS spectra of 8 . The ${ }^{29} \mathrm{Si}$

(18) Mason, J., Ed. Multinuclear NMR; Plenum Press: New York, London, 1987.

(19) (a) Mehring, M. Principles of High Resolution NMR in Solids; Springer-Verlag: Berlin, 1983. (b) Wind, R. A.; Dec, S. F.; Lock, H. Maciel, G. E. J. Magn. Reson. 1988, 79, 136-139. resonances of both 7 and 8 show similar half-height line widths under ${ }^{19} \mathrm{~F}$ high-power decoupling $(7: 350 \mathrm{~Hz} ; 8: 300 \mathrm{~Hz}$ ). As mentioned before, the two ${ }^{29} \mathrm{Si}$ resonances for 7 which one would expect on the basis of the single-crystal X-ray diffraction studies 10 are not resolved. Line widths of the order of $300 \mathrm{~Hz}$ certainly have to be considered substantial for ${ }^{29}$ Si MAS or CP MAS spectra of crystalline silicates. In the case of compounds 7 and 8 it may only seem too tempting to ascribe this broadening entirely to some residual ${ }^{1} \mathrm{H}-{ }^{29} \mathrm{Si}$ dipolar interactions. ${ }^{20}$ However, also for 8 the cross polarization efficiency for the ${ }^{19} \mathrm{~F} \rightarrow 29 \mathrm{Si} \mathrm{CP}$ MAS experiment is fairly poor as may be seen in Figure 4. In the absence of motion ${ }^{19} \mathrm{~F} \rightarrow{ }^{29} \mathrm{Si}$ cross polarization has been shown to be a highly efficient process ${ }^{16,21}$ so that it seems reasonable to postulate a certain degree of $\mathrm{SiF}_{4}$ nonrigidity also for compound 8. Another indication for the presence of such $\mathrm{SiF}_{4}$ nonrigidity in solid 7 and 8 can be obtained from the respective ${ }^{1} \mathrm{H} \rightarrow{ }^{29} \mathrm{Si}$ $\mathrm{CP}$ MAS spectra. 7 yields a ${ }^{~} \mathrm{H} \rightarrow{ }^{29} \mathrm{Si} \mathrm{CP}$ MAS resonance $\left(\nu_{1 / 2}\right.$ $=350 \mathrm{~Hz}$, excellent $\mathrm{CP}$ efficiency with $\mathrm{CP}$ contact times of approximately $5 \mathrm{~ms}$ ) which displays no fine structure due to the interplay of scalar and dipolar ${ }^{19} \mathrm{~F} /{ }^{29} \mathrm{Si}$ interactions. We may thus speculate that motional processes in solid 7 at room temperature should be restricted to the $\mathrm{SiF}_{4}$ moiety, leaving the ${ }^{1} \mathrm{H}-{ }^{29} \mathrm{Si}$ interactions rather undisturbed. In fact, exchange processes similar to those shown for related ionic compounds in solution (see for example refs $l c, e, f$ and $2 i, m, n, t)$, would be fully in agreement with our various CP MAS findings.

Finally, it should be mentioned that the isotropic ${ }^{29} \mathrm{Si}$ chemical shifts of $7\left(-121.1 \mathrm{ppm}\right.$; in $\left.\mathrm{CD}_{3} \mathrm{CN}-122.9 \mathrm{ppm}^{10}\right)$ and $8(-112.4$ $\mathrm{ppm}$ ) are in good agreement with the existence of pentacoordinate silicon in these zwitterions.

For compounds 9 and 10 we can only report a complete failure to obtain either ${ }^{29} \mathrm{Si}$ MAS spectra with ${ }^{19} \mathrm{~F}$ high-power decoupling or ${ }^{19} \mathrm{~F} \rightarrow{ }^{29} \mathrm{Si}$ CP MAS spectra. Using similar experimental parameters as for 7 and 8 to record the ${ }^{29} \mathrm{Si}$ ( ${ }^{19} \mathrm{~F}$ decoupled) MAS spectra (i.e. spinning rates of $4-5 \mathrm{kHz},{ }^{29} \mathrm{Si}$ pulse widths of $30^{\circ}$, and recycle delays of $30-60 \mathrm{~s}$ ) yielded no detectable ${ }^{29} \mathrm{Si}$ resonances for 9 and 10. Various ${ }^{19} \mathrm{~F} \rightarrow 29 \mathrm{Si} \mathrm{CP} \mathrm{MAS} \mathrm{experiments} \mathrm{on} 9$ and 10 were equally unsuccessful. We have no reason to believe that the ${ }^{29} \mathrm{Si}$ longitudinal relaxation times $T_{1}$ for 9 and 10 could be much longer than for 7 and 8 . Therefore, similarly conservative pulse conditions (i.e. small ${ }^{29} \mathrm{Si}$ flip angles and rather long relaxation delays) as used successfully for 7 and 8 should yield reasonable ${ }^{29} \mathrm{Si}$ MAS spectra for 9 and 10 . We have to come to the conclusion that for both 9 and 10 the ${ }^{29}$ Si resonances (even under ${ }^{19} \mathrm{~F}$ high-power decoupling conditions) at room temperature are broadened beyond detection. Again, this could be a combined effect of substantial residual ${ }^{1} \mathrm{H}-29 \mathrm{Si}$ dipolar interactions (more for $\mathbf{9}$ and $\mathbf{1 0}$ than for $\mathbf{7}$ and $\mathbf{8}$ ) and/or motional processes which would have to be in the coalescence regime near room temperature in order to broaden the ${ }^{29} \mathrm{Si}$ resonances of 9 and 10 to such an extent.

Acknowledgment. Support of this work by the Deutsche Forschungsgemeinschaft, the Fonds der Chemischen Industrie, Wacker-Chemie GmbH (Burghausen, Germany) and Bayer AG (Leverkusen and Wuppertal-Elberfeld, Germany) is gratefully acknowledged.

Supplementary Material Avalable: For 9 and 10, respectively, anisotropic thermal parameters (Tables S1 and S4), hydrogen atom parameters (Tables $S 2$ and SS), and additional bond angles and distances (Tables S3 and S6) (6 pages). Ordering information is given on any current masthead page.

(20) No ${ }^{1} \mathrm{H}$ high-power decoupling in addition to the ${ }^{19} \mathrm{~F}$ bigh-power decoupling could be used as this would require a triply-tuned probe circuit: our probe is only doubly-tuned and matched for ${ }^{19} \mathrm{~F} / \mathrm{X}$-nucleus frequencies. Accordingly, ' $\mathrm{H} \rightarrow{ }^{29} \mathrm{Si}$ CP MAS experiments had to be carried out in the absence of ${ }^{19} \mathrm{~F}$ high-power decoupling.

(21) Schaller, T.; Dingwell, D. B.; Keppler, H.; Knoller, W.; Merwin, L. H. Sebald, A. Geochim. Cosmochim. Acta 1992, 56, 701-707. 